\title{
Power Corrections for Jets at Hadron Colliders
}

\author{
Matteo Cacciari $^{1}$, Mrinal Dasgupta ${ }^{2}$, Lorenzo Magnea $^{3}$ and Gavin Salam ${ }^{1}$ \\ 1- LPTHE, CNRS UMR 7589, Université P. et M. Curie (Paris 6) \\ Université D. Diderot (Paris 7), 75252 Paris Cedex 05, France \\ 2- School of Physics and Astronomy, University of Manchester \\ Oxford Road, Manchester M13 9PL, U.K. \\ 3- Dipartimento di Fisica Teorica, Università di Torino, and \\ INFN, Sezione di Torino, Via P. Giuria, I-10125 Torino, Italy
}

\begin{abstract}
We discuss non-perturbative QCD corrections to jet distributions in hadron collisions, focussing on hadronisation and underlying event contributions. Using soft gluon resummation and Monte-Carlo modelling we show that hadronisation dominates at small values of the jet radius $R$, behaving as $1 / R$, while underlying event corrections grow with the jet area. This provides a handle to disentangle them and parametrize them in terms of measurable QCD parameters, which might enjoy a degree of universality.
\end{abstract}

\section{Introduction}

With the advent of the LHC particle physics will once again break into new territory at the high energy frontier. With $14 \mathrm{TeV}$ available in the center of mass, one would naively expect that the dynamics of confinement and low-energy QCD would decouple and, thanks to factorization, have a minimal influence on high- $p_{\perp}$ observables (a typical figure of merit being $\left.\Lambda / p_{\perp} \sim \mathcal{O}\left(10^{-3}\right)\right)$. In general, this expectation is not fulfilled. Even at very high energy, for example, no hadronic cross section can be precisely determined without a detailed knowledge of parton distributions in the colliding hadrons. Furthermore, a wide range of observables of interest for both Standard Model and BSM physics must rely upon a definition of hadronic jets and a measurement of the jet energy scale. Experience gained at the Tevatron [2] shows, for example, that a $1 \%$ uncertainty in the jet energy scale causes a $1 \mathrm{GeV}$ uncertainty in the top quark mass determination, and is reflected in a $10 \%$ uncertainty in the single inclusive jet $p_{\perp}$ distribution at $p_{\perp} \sim 500 \mathrm{GeV}^{\mathrm{a}}$.

Assuming a jet has been reconstructed with an infrared and collinear safe algorithm, dependent upon a parameter $R$ defining its size in the rapidity-azimuth plane, the energy of the jet will differ from the energy of the hard parton that originated it because of a variety of physical phenomena. Radiation from the underlying event and from pileup will spill inside the jet cone, increasing its measured energy; on the other hand, radiation produced during hadronisation will spill outside the jet cone, leading to a negative correction. It is important to realize that, while some of these corrections are definitely outside the reach of perturbative calculations (this is certainly the case for pileup, and to a certain extent for the underlying event), hadronisation corrections can be explored with perturbative methods, supplemented by soft gluon resummation and power correction technology. These methods have been successfully applied to simpler processes such as $e^{+} e^{-}$annihilation and DIS (for a review, see [4]), where studies of event shapes showed that power corrections to distributions can be efficiently parametrized in terms of a limited number of non-perturbative parameters, enjoying a remarkable degree of universality $[5,6]$. Here we will apply for the first time these

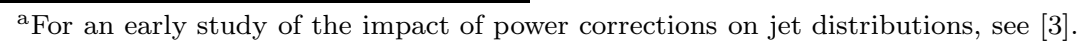


methods to jet distributions in hadron collisions, a much more challenging environment. We consider, as an example, the single inclusive jet $p_{\perp}$ distribution: we show that perturbative methods lead to a prediction for the $R$ dependence of the leading power correction, which turns out to be singular (behaving as $1 / R$ ) for hadronisation corrections, while, as might be expected, it grows as $R^{2}$ for the underlying event. We go on to compare the analytic result to Monte Carlo models, finding broad agreement as well as some interesting differences in the details.

\section{Issues in soft gluon resummation for jet distributions}

Soft gluon resummation for the single-inclusive jet $p_{\perp}$ distribution was first performed in [7], using techniques developed in $[8]$ and $[9]^{\mathrm{b}}$. Since jet production in hadron collisions generically involves at least four colored partons, this is the first case in which nontrivial mixing of colour structures occurs. Formally, the structure of the resummed cross section is

$$
E_{J} \frac{d^{3} \sigma}{d^{3} p_{J}}=\frac{1}{s} \exp \left[\sum_{p=1}^{2} \mathcal{E}_{\mathrm{IN}}^{(p)}+\sum_{p=J, R} \mathcal{E}_{\mathrm{OUT}}^{(p)}\right] \cdot \operatorname{Tr}[H S] .
$$

The factors $\mathcal{E}_{\text {IN }}$ and $\mathcal{E}_{\text {OUT }}$ exponentiate collinear logarithms associated with initial state radiation of the colliding partons, and with the measured and the recoil outgoing jets, respectively. At the level of power corrections, these factors are expected to generate contributions of order $\left(\Lambda / p_{\perp}\right)^{2}$, associated with jet mass effects. These corrections are negligible, and therefore we will concentrate on the contributions of soft gluons emitted at wide angles from the jet, embodied in the last factor in Eq. (1). Here the trace is taken in the space of representations of the color group that can be constructed out of the scattering hard partons, while $H$ and $S$ are matrices containing hard and soft gluon contributions respectively. The exponentiation of soft radiation and the structure of color mixing can be simply understood [11] by resorting to the eikonal approximation. One can show that all soft logarithms can be organized in terms of eikonal colored dipoles, given by

$$
\mathcal{D}_{i j}\left(Q, Q_{0}\right) \equiv \int_{Q_{0}}^{Q} d \kappa_{\perp}^{(i j)} \kappa_{\perp}^{(i j)} \alpha_{s}\left(\kappa_{\perp}^{(i j)}\right) \int d \eta \frac{d \phi}{2 \pi} \frac{p_{i} \cdot p_{j}}{p_{i} \cdot k p_{j} \cdot k} T_{i} \cdot T_{j}
$$

where the indices $i, j$ label hard partons, $T_{i}, T_{j}$ are the color generators in the corresponding representation, and $\kappa_{\perp}^{(i j)}=2 p_{i} \cdot k p_{j} \cdot k /\left(p_{i} \cdot p_{j}\right)$ is the transverse momentum with respect to the emitting dipole. For $n<4$ hard partons, all products of color matrices can be expressed in terms of Casimir operators, and thus there is no mixing of color structures.

Before extending the discussion to power corrections, it should be noted that usage of resummations such as Eq. (1) requires great care both in the choice and in the definition of the observable. In general jet cross sections, which involve an explicit slicing of phase space, are affected by nonglobal logarithms [12]. In the present case, it turns out that the $p_{\perp}$ distribution is a global observable. Nonglobal logarithms will generically spoil Eq. (1) at NLL level, and might influence power correction in a way which is not currently understood. One should also note that the choice of jet algorithm may affect the results quite drastically: IR safety is a must; furthermore, we will work assuming that the jet momentum is reconstructed using four-momentum recombination.

\footnotetext{
${ }^{\mathrm{b}} \mathrm{A}$ refinement of the implementation of Ref. [7] was recently proposed in [10]
} 


\section{Radius dependence of power corrections}

In order to analyze the structure of power corrections using the resummation, we consider separately each dipole in Eq. (2). For the sake of simplicity we place the measured jet at zero rapidity, which does not qualitatively affect our results. Let $\delta \xi^{ \pm}\left(k_{\perp}, \eta, \phi\right)$ be the contribution of a soft gluon with momentum $k$ to the observable, which we normalize as $\xi \equiv 1-p_{\perp} / \sqrt{S}$. Note that the contribution is different if the gluon is recombined with the jet $\left(\delta \xi^{+}\right)$, or is left out of it $\left(\delta \xi^{-}\right)$. We then construct the shift in the $\xi$ distribution due to the $(i j)$ dipole by integrating $\delta \xi^{ \pm}\left(k_{\perp}, \eta, \phi\right)$ over the gluon phase space, with a measure given by the eikonal dipole,

$$
\Delta \xi_{i j}^{ \pm}(R) \equiv \int_{ \pm} d \eta \frac{d \phi}{2 \pi} \int_{0}^{\mu_{F}} d \kappa_{\perp}^{(i j)} \alpha_{s}\left(\kappa_{\perp}^{(i j)}\right) k_{\perp}\left|\frac{\partial k_{\perp}}{\partial \kappa_{\perp}^{(i j)}}\right| \frac{p_{i} \cdot p_{j}}{p_{i} \cdot k p_{j} \cdot k} \delta \xi^{ \pm}\left(k_{\perp}, \eta, \phi\right) .
$$

Note that $\delta \xi^{+}$is integrated inside the jet cone, and $\delta \xi^{-}$outside of it. The result is then a function of the jet radius $R$.

Different dipoles give different $R$ dependences, with a transparent physical interpretation. The dipole constructed out of the two incoming partons has a leading power correction growing like $R^{2}$, and it is natural to interpret it as the way in which the buildup of the underlying event begins to be seen from perturbation theory. Dipoles involving the measured jet, on the other hand, behave as $1 / R$ for small $R$. This behavior arises from gluons which are not recombined with the jet: at small $R$, they are allowed to be emitted very close in phase space to the radiating parton, and they begin to see the corresponding collinear singularity. All dipole integrals are expressed in terms of a low-energy moment of the strong coupling, $\mathcal{A}\left(\mu_{F}\right)$, which in principle could be related to the analogous quantity measured in $e^{+} e^{-}$annihilation. As an example, we consider the contribution of the $q q \rightarrow q q$ channel to the $p_{\perp}$ distribution. The dipole comprising the two incoming quarks gives

$$
\Delta \xi_{\text {in }- \text { in }}(R)=-\frac{4}{\sqrt{S}} \mathcal{A}\left(\mu_{F}\right) R J_{1}(R)=-\frac{1}{\sqrt{S}} \mathcal{A}\left(\mu_{F}\right)\left(2 R^{2}-\frac{1}{4} R^{4}+\mathcal{O}\left(R^{6}\right)\right) .
$$

The dipole involving an incoming leg and the measured jet, on the other hand, gives

$$
\Delta \xi_{\text {in }-\mathrm{J}}(R)=\frac{1}{\sqrt{S}} \mathcal{A}\left(\mu_{F}\right)\left(\frac{4}{R}-\frac{5}{4} R+\frac{23}{768} R^{3}+\mathcal{O}\left(R^{5}\right)\right) .
$$

Notice that, as expected, this corresponds to a negative shift in the $p_{\perp}$ distribution, given the definition of $\xi$. We remark also that all singular contributions are essentially abelian in nature and can be directly collected into an overall shift in the physical distribution, weighed by $C_{F}\left(C_{A}\right)$ for a quark- (gluon-) initiated jet. Terms subleading in $R$, however, including Eq. (4), must be recombined taking color mixing into account, and their contribution is more intricate than a simple shift.

\section{Monte-Carlo analysis of hadronisation and underlying event}

A powerful cross-check of the validity of the above discussion is provided by examining hadronisation corrections for a range of jet algorithms in Monte Carlo simulations. We consider dijet events from both Pythia [13] and Herwig [14] and select events whose highest$p_{\perp}$ jet at parton-level has $55<p_{\perp}<70 \mathrm{GeV}$. The selection at parton level is intended 

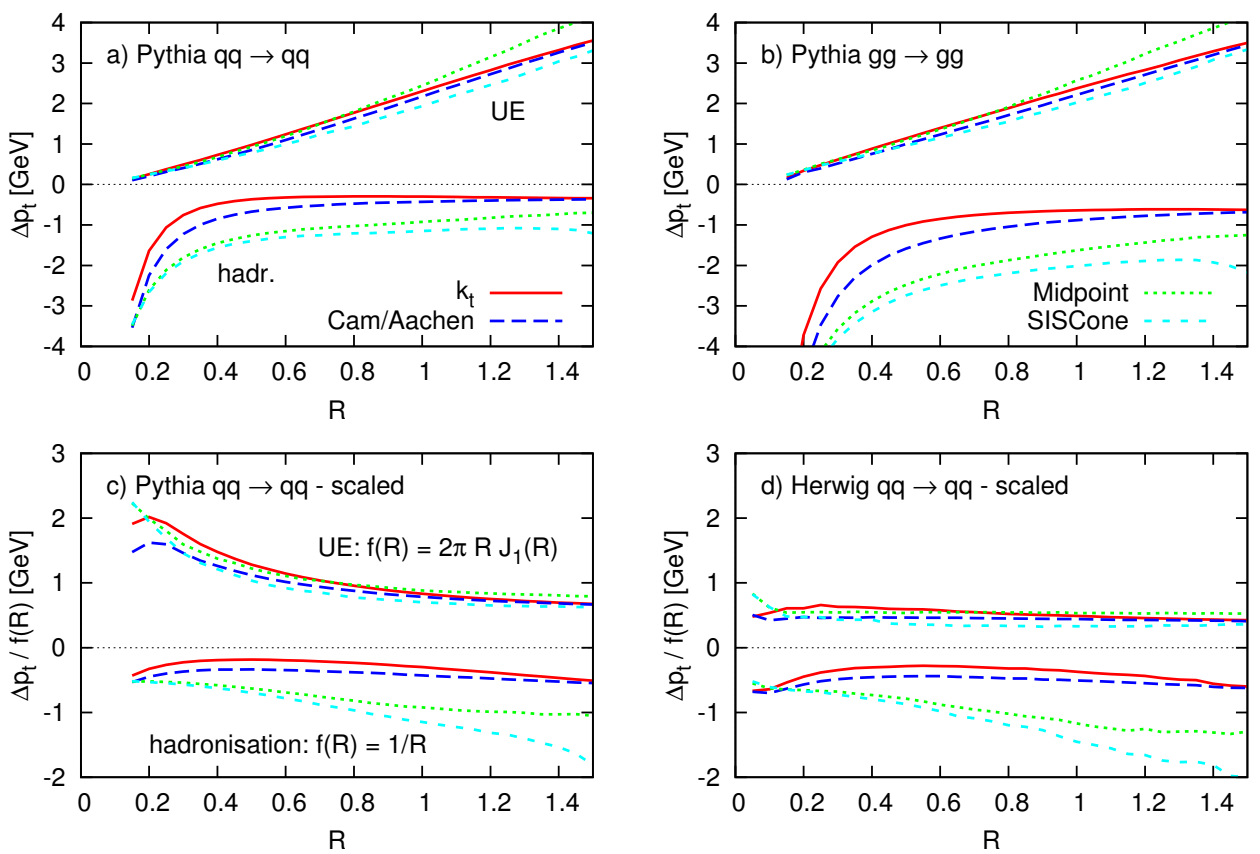

Figure 1: Shifts in $p_{t}$ associated with hadronisation and underlying-event contributions for the two leading jets in Pythia 6.410 and Herwig 6.510 dijet events.

to eliminate selection bias associated with non-perturbative effects, as is appropriate for comparison with our analytical formulae. For each event we examine the average difference in $p_{t}$ for the two leading jets at hadron-level as compared to parton level. This difference is separated into a hadronisation component and an underlying event contribution, the latter 'defined' as that obtained when switching on underlying event (UE) and/or multipleinteractions in each Monte Carlo program, using the default parameters of each. The UE contribution is expected to be uniform in rapidity and azimuth, scaling as $\pi R^{2}$ for small $R$, or as $2 \pi R J_{1}(R)$ for general $R$ with $E$-scheme (4-momentum) recombination.

The upper plots of Fig. 1 show the two non-perturbative components, for two hard scattering channels, as a function of $R$ for the $k_{t}$ [15], Cambridge/Aachen [16], Midpoint ${ }^{\mathrm{c}}$ cone [17] and SISCone [18] jet algorithms (all run through FastJet [19]). The negative result for hadronisation, divergent at small $R$, is consistent with Eq. (5). The rough factor of two between the hadronisation corrections for the $q q \rightarrow q q$ and $g g \rightarrow g g$ is consistent with the $C_{A} / C_{F}$ ratio expected from Sect. 3. One notes that the cone-type algorithms have more negative corrections, differing from the sequential recombination algorithms by a term roughly independent of $R$ whose explanation is beyond the scope of the single-gluon calculation given above. The UE event contribution to the jet $p_{\perp}$ is positive, as expected, and for the typical range of $R$ studied experimentally, $0.4<R<0.7$, similar in magnitude to the hadronisation correction. It is largely identical for all algorithms, and roughly independent

\footnotetext{
"This algorithm is infrared unsafe and should be thought of as a "legacy" algorithm, shown only for historical purposes.
} 
of the hard scattering channel.

The analytical $R$ dependence can be studied in more depth by scaling out the expectations for hadronisation and UE, as is shown in the lower plots of Fig. 1. For recombination algorithms the result is roughly independent of $R$, as expected from Eq. (5). The normalisation of $\sim 0.5 \mathrm{GeV}$ is consistent with the magnitude of hadronisation corrections extracted at LEP. In contrast, the normalised UE correction is not constant in $R$. This can be shown to be a consequence of Pythia's implementation of colour-reconnections between the hard partons and the underlying event and when examining models without such reconnections, such as Herwig (and also Jimmy [20]), Fig. 1d, this effect disappears. One notes that if one extracts the UE $p_{\perp}$ density per unit rapidity (in the figure it is normalised per unit area) then it is $\sim 6$ times larger than the similarly normalised hadronisation correction, suggestive of far more violent non-perturbative dynamics.

\section{Acknowledgements}

We are grateful to Torbjorn Sjöstrand, Peter Skands and Jon Butterworth for assistance and helpful comments. Work supported in part by the EU Research and Training Network 'HEPTOOLS' under contract MRTN-CT-2006-035505 and by grant ANR-05-JCJC-0046-01 from the French Agence Nationale de la Recherche.

\section{References}

[1] Slides: http://indico. cern.ch/contributionDisplay. py? contribId=179\&sessionId=6\&conf $I d=9499$

[2] A. Bhatti et al., Nucl. Instrum. Meth. A 566375 (2006), arXiv:hep-ex/0510047.

[3] M.L. Mangano, arXiv:hep-ph/9911256.

[4] M. Dasgupta and G. P. Salam, J. Phys. G 30 R143 (2004), arXiv;hep-ph/0312283.

[5] C. F. Berger and G. Sterman, JHEP 0309058 (2003), arXiv:hep-ph/0307394.

[6] C.F. Berger and L. Magnea, Phys. Rev. D 70094010 (2004), arXiv:hep-ph/0407024.

[7] N. Kidonakis and J. F. Owens, Phys. Rev. D 63054019 (2001), arXiv:hep-ph/0007268.

[8] E. Laenen, G. Oderda and G. Sterman, Phys. Lett. B 438173 (1998), arXiv:hep-ph/9806467.

[9] N. Kidonakis, G. Oderda and G. Sterman, Nucl. Phys. B 525299 (1998), arXiv:hep-ph/9801268.

[10] D. de Florian and W. Vogelsang, arXiv:0704.1677 [hep-ph].

[11] Yu. L. Dokshitzer and G. Marchesini, JHEP 0601007 (2006), arXiv:hep-ph/0509078.

[12] M. Dasgupta and G. P. Salam, Phys. Lett. B 512323 (2001), arXiv:hep-ph/0104277.

[13] T. Sjostrand et al., Comp. Phys. Comm. 135238 (2001), arXiv:hep-ph/0010017; arXiv:hep-ph/0308153.

[14] G. Marchesini et al., Comp. Phys. Comm. 67465 (1992); G. Corcella et al., JHEP 0101010 (2001), arXiv:hep-ph/0011363.

[15] S. Catani et al., Nucl. Phys. B 406187 (1993); S.D. Ellis and D.E. Soper, Phys. Rev. D 483160 (1993), arXiv:hep-ph/9305266.

[16] Y.L. Dokshitzer et al., JHEP 9708001 (1997), arXiv:hep-ph/9707323; M. Wobisch and T. Wengler, arXiv:hep-ph/9907280.

[17] G. C. Blazey et al., arXiv:hep-ex/0005012.

[18] G.P. Salam and G. Soyez, JHEP 0705086 (2007), arXiv:0704.0292

[19] M. Cacciari and G. P. Salam, Phys. Lett. B 64157 (2006), arXiv:hep-ph/0512210.

[20] J. M. Butterworth, J. R. Forshaw and M. H. Seymour, Z. Phys. C 72 (1996) 637, arXiv:hep-ph/9601371. 